\title{
A Fresh Look To Exact Solutions of Some Coupled Equa- tions
}

\author{
Berat Karaagac ${ }^{1, *}$ Nuri Murat Yagmurlu ${ }^{2, * *}$ Alaattin Esen ${ }^{2, * * *}$ and Selcuk Kutluay ${ }^{2, * * *}$ \\ ${ }^{1}$ Adiyaman University, Department of Mathematics Education,Adiyaman, TURKEY \\ ${ }^{2}$ Inonu University, Department of Mathematics, Malatya, TURKEY
}

\begin{abstract}
This manuscript is going to seek travelling wave solutions of some coupled partial differential equations with an expansion method known as SineGordon expansion method. Primarily, we are going to employ a wave transformation to partial differential equation to reduce the equations into ordinary differential equations. Then, the solution form of the handled equations is going to be constructed as polynomial of hyperbolic trig or trig functions. Finally, with the aid of symbolic computation, new exact solutions of the partial differentials equations will have been found.
\end{abstract}

\section{Introduction}

The exact solutions of both ordinary and partial differential equations help scientists to comprehend the physical mechanism of the natural phenomena clearly. Thus, being able to obtain the exact solutions of several equations including but not limited to solitary wave solutions has become one of the main concerns of many scientists. For the sake of obtaining exact solutions, several methods and techniques have been developed can be seen in Refs. [1-7].

In the present study, we are going to seek the exact solutions of variant Boussinesq equation and coupled Klein Gordon equation via Sine-Gordon expansion method [8, 9]. Those equations have been solved by several authors using different methods and techniques in [10] and therein.

\section{Description of the Sine-Gordon expansion method}

In this section, we will introduce Sine-Gordon expansion method which is a well known and popular method $[11,12]$. Let us take into consideration the following widely known Sine-Gordon equation

$$
u_{x x}-u_{t t}=m^{2} \sin u
$$

where $u=u(x, t)$ is the desired function and $m$ is a constant. When the transformation $u(x, t)=U(\xi)$ in which $\xi=k x+l t$ is introduced, the Eq. (1) is reduced to a nonlinear

\footnotetext{
*e-mail: bkaraagac@adiyaman.edu.tr

**e-mail: murat.yagmurlu@inonu.edu.tr

***e-mail: alaattin.esen@inonu.edu.tr

****e-mail: selcuk.kutluay@inonu.edu.tr
} 
ordinary differential equation given in the following form

$$
U^{\prime \prime}=\frac{m^{2}}{k^{2}-l^{2}} \sin (U)
$$

When the both sides of Eq. (2) is multiplied by $U^{\prime}$ and integrated once, the following form is obtained

$$
\left[\left(\frac{U}{2}\right)^{\prime}\right]=\frac{m^{2}}{k^{2}-l^{2}} \sin ^{2}\left(\frac{U}{2}\right)+K
$$

in which the integration constant is denoted by $K$. When $K=0, \frac{U}{2}=w(\xi)$, and $a^{2}=\frac{m^{2}}{k^{2}-l^{2}}$ are taken, the new form of the equation (3) is obtained as follows

$$
w^{\prime}=a \sin (w)
$$

When $a=1$ is taken, the Eq. (4), the most fundamental form of Eq. (1) is finally obtained as follows

$$
w^{\prime}=\sin (w)
$$

It is obvious that the Eq. (5) is obtained from the original Sine-Gordon equation by some simplification processes. Now, Eq. (5) can be solved more easily than the original SineGordon equation and its solutions can be obtained as follows

$$
\sin (w)=\sin (w(\xi))=\left.\frac{2 p e^{\xi}}{p^{2} e^{2 \xi}+1}\right|_{p=1}=\sec h(\xi), \quad \cos (w)=\cos (w(\xi))=\left.\frac{p^{2} e^{2 \xi}-1}{p^{2} e^{2 \xi}+1}\right|_{p=1}=\tanh (\xi)
$$

where the integration constant is taken as $p \neq 0$. It is high time to generalize this approach to a nonlinear partial differential equation given as

$$
F\left(u, u_{x}, u_{t}, u_{x t}, u_{t t}, \ldots\right)=0 .
$$

When, again, the transformation $u(x, t)=U(\xi)$ in which $\xi=k x+l t$ is introduced, Eq. (7) is converted into the following nonlinear ordinary differential equation

$$
G\left(U, U^{\prime}, U^{\prime \prime}, \ldots\right)=0
$$

In a similar way followed above, it is assumed that the solution $U(\xi)$ of the nonlinear equation given in Eq. (8) can be formed as follows

$$
U(\xi)=\sum_{i=1}^{N} \tanh ^{i-1}(\xi)\left[B_{i} \sec h(\xi)+A_{i} \tanh (\xi)\right]+A_{0}
$$

When the Eqs. (6) are taken into consideration, Eq. (9) can also be written as follows

$$
U(w)=\sum_{i=1}^{N} \cos ^{i-1}(w)\left[B_{i} \sin (w)+A_{i} \cos (w)\right]+A_{0}
$$

When the value of $N$ is determined via the homogenous balance principle, Eq. (10) is substituted into Eq. (8) and they are compared term by term; a nonlinear algebraic system is acquired. Solving this new system leads to obtain the travelling wave solutions of Eq. (7). 


\section{Applications}

\subsection{Variant Boussinesq equation}

Let us consider the variant Boussinesq equations handled by Wang [13] in 1995 in the following form

$$
u_{t+}(u v)_{x}+v_{x x x}=0, \quad v_{t}+u_{x}+v v_{x} .=0
$$

In this system, subscripts $t$ and $x$ denote partial derivatives with respect to time and space, respectively. In Eq. (11) when

$$
u(x, t)=u(\xi), \quad v(x, t)=v(\xi)
$$

are taken, where $\xi=\alpha x-c t$, under these conditions, the following equations are obtained

$$
-c u^{\prime}+\alpha(u v)^{\prime}+\alpha^{3} v^{\prime \prime \prime}=0, \quad-c v^{\prime}+\alpha u^{\prime}+\alpha v v_{x}=0 .
$$

By integrating the equations in Eq. (13) once, the following equations are found

$$
-c u+\alpha u v+\alpha^{3} v^{\prime \prime}=r, \quad-c v+\alpha u+\frac{\alpha}{2} v^{2}=s_{1}
$$

where $r$ and $s_{1}$ are integration constants. When $u=\frac{c}{\alpha} v-\frac{1}{2} v^{2}+\frac{s_{1}}{\alpha}$ are taken, and $s=s_{1} / \alpha$ is assumed, the following equation is obtained. When the some required simplifications are made, we obtain the following equations

$$
\alpha^{3} v^{\prime \prime}-\frac{\alpha}{2} v^{3}+\frac{3 c}{2} v^{2}+\left(\alpha s_{1}-\frac{c^{2}}{\alpha}\right) v-\left(c s_{1}+r\right)=0 .
$$

Then using the homogenous balance condition we obtain, $N=1$. Thus, the solution is formed as

$$
u(\xi)=\frac{c}{\alpha} v(\xi)-\frac{1}{2} v(\xi)^{2}+\frac{s_{1}}{\alpha}, \quad v(\xi)=A_{0}+A_{1} \tanh (\xi)+B_{1} \sec h(\xi)
$$

Substituting the solution given above in reduced equation given in Eq. (13), collecting the coefficient power of $\tanh (\xi), \sec h(\xi)$ and $\sec h(\xi) \tanh ^{i}(\xi)$ then equalizing each of coefficient to zero, we get the following algebraic equation systems in terms of the parameters $A_{0}, A_{1}, B_{1}, c, s, r$ and $\alpha$

$$
\begin{aligned}
& (\sec h(\xi) \tanh (\xi))^{0}:-r-\frac{c^{2} A_{0}}{\alpha}+\frac{3 c A_{0}^{2}-\alpha A_{0}^{3}+3 c B_{1}^{2}-3 \alpha A_{0} B_{1}^{2}}{2}-c s_{1}+\alpha A_{0} s_{1}, \quad(\tanh (\xi))^{3}: 2 \alpha^{3} A_{1}-\frac{\alpha A_{1}^{3}}{2}+\frac{3 \alpha A_{1} B_{1}^{2}}{2} \\
& (\sec h(\xi))^{1}:-\frac{c^{2} B_{1}}{\alpha}-\alpha^{3} B_{1}+3 c A_{0} B_{1}-\frac{3 \alpha A_{0}^{2} B_{1}-\alpha B_{1}^{3}}{2}+\alpha B_{1} s_{1}, \quad(\sec h(\xi) \tanh (\xi)): 3 c A_{1} B_{1}-3 \alpha A_{0} A_{1} B_{1} \\
& (\tanh (\xi))^{1}: \frac{-c^{2} A_{1}}{\alpha}-2 \alpha^{3} A_{1}+3 c A_{0} A_{1}-\frac{3 \alpha A_{0}^{2} A_{1}+3 \alpha A_{1} B_{1}^{2}}{2}+\alpha A_{1} s_{1}, \quad\left(\sec h(\xi) \tanh ^{2}(\xi)\right): \alpha^{3} B_{1}-\frac{3}{2} \alpha A_{1}^{2} B_{1} \\
& (\tanh (\xi))^{2}: \frac{3 c A_{1}^{2}-3 \alpha A_{0} A_{1}^{2}-3 c B_{1}^{2}+3 \alpha A_{0} B_{1}^{2}}{2}+\alpha^{3} B_{1}+\frac{\alpha B_{1}^{3}}{2} .
\end{aligned}
$$

Solving the equation system simultaneously, we get the required coefficients for constructing desirable solution as follows:

$$
\begin{aligned}
& \left\{\begin{array}{l}
\left.A_{1}=\mp 2 \alpha, B_{1}=0, c=\alpha A_{0}, s=\frac{1}{2}\left(4 \alpha^{2}-A_{0}^{2}\right), r=\frac{1}{2}\left(4 \alpha^{3} A_{0}-\alpha A_{0}^{3}-2 \mathrm{cs}_{1}\right)\right\} \\
\left.A_{1}=0, B_{1}=2 \mp i \alpha, c=\alpha A_{0}, s=\frac{1}{2}\left(-2 \alpha^{2}-A_{0}^{2}\right), r=\frac{1}{2}\left(-2 \alpha^{3} A_{0}-\alpha A_{0}^{3}-2 \mathrm{cs}_{1}\right)\right\}
\end{array}\right. \\
& \left\{\begin{array}{l}
A_{1}=\mp \alpha, B_{1}=-i \alpha, c=\alpha A_{0}, s=\frac{\alpha^{2}-A_{0}^{2}}{2}, r=\frac{-2 c s+\alpha^{3} A_{0}-\alpha A_{0}^{3}}{2}
\end{array}\right\}
\end{aligned}
$$

and the solution is formed as

$$
\begin{aligned}
& u_{1}(x, t)=2 \alpha^{2} \operatorname{sech}^{2}\left(\alpha\left(x-A_{0} t\right)\right), \quad v_{1}(x, t)=A_{0} \mp 2 \alpha \tanh \left(\alpha\left(x-A_{0} t\right)\right) \\
& u_{2}(x, t)=\alpha^{2}\left(2 \operatorname{sech}^{2}\left(\alpha\left(x-A_{0} t\right)\right)-1\right), \quad v_{2}(x, t)=A_{0} \mp 2 i \alpha \operatorname{sech}\left(\alpha\left(x-A_{0} t\right)\right) \\
& u_{3}(x, t)=\frac{i \alpha^{2}}{i \mp \sinh \left(\alpha\left(x-t A_{0}\right)\right)}, \quad v_{3}(x, t)=A_{0} \mp \alpha \tanh \left(x \alpha-\alpha A_{0} t\right)-i \alpha \sec h\left(x \alpha-\alpha A_{0} t\right)
\end{aligned}
$$




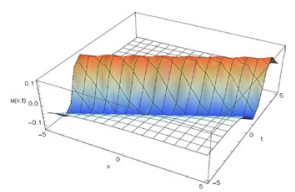

(a)

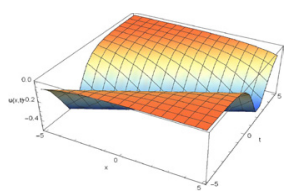

(c)

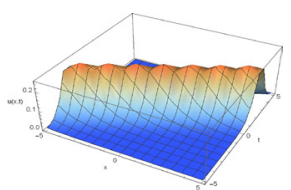

(b)

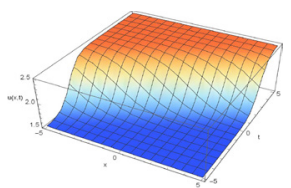

(d)

Figure 1: Solution of Variant Boussinesq equation for $\alpha=c=0.5, A_{0}=2$

Additionally, the graphical representation of $u_{3}$ and $v_{3}$ is illustrated in Figure 1 for selected values of some parameters. In the literature; different types of solutions can be found $[10,14]$ and there in. However, within the knowledge of the authors, $u_{3}$ and $v_{3}$ solutions are new exact solutions of the Variant Boussinesq equation.

\subsection{Coupled Klein Gordon equation}

Now, we are going to consider coupled Klein Gordon equation in the form

$$
u_{x x}-u_{t t}-u+2 u^{3}+2 u v=0, \quad v_{x}-v_{t}-4 u u_{t}=0
$$

where $u(x, t)=u(\xi)$ and $v(x, t)=v(\xi)$ and $\xi=\alpha x-c t$. After these assumptions we can write the following equations,

$$
\alpha^{2} u^{\prime \prime}-c^{2} u^{\prime \prime}-u+2 u^{3}+2 u v=0, \quad \alpha v^{\prime}+c v^{\prime}+4 c u u^{\prime}=0
$$

If we take the integral of the second equation and make some arrangements, we obtain $v=$ $\frac{r-2 c u^{2}}{(\alpha+c)}$ where $r$ is an integral constant. If we write this newly obtained equation in the first equation in Eq. (17), we obtain $\left(\alpha^{2}-c^{2}\right) u^{\prime \prime}+\left(2-\frac{4 c}{\alpha+c}\right) u^{3}+\left(\frac{2 r}{\alpha+c}\right) u=0$. Then using the homogenous balance condition, we obtain $n=1$. Following the same procedure presented in the first example, we get algebraic equations after solving the system, required coefficients are given, respectively, by

$$
\left\{\begin{array}{l}
\left.A_{0}=0, A_{1}=-i(\alpha+c), B_{1}=0, r=\frac{1}{2}\left(2 \alpha^{3}+\alpha-2 c^{3}-2 \alpha c^{2}+2 \alpha^{2} c+c\right)\right\} \\
\left.A_{0}=0, A_{1}=0, B_{1}=-\alpha-c, r=\frac{1}{2}\left(-\alpha^{3}+\alpha+c^{3}+\alpha c^{2}-\alpha^{2} c+c\right)\right\} \\
\left.A_{0}=0, A_{1}=\frac{1}{2} i(\alpha+c), B_{1}=\frac{1}{2}(-\alpha-c), r=\frac{1}{4}\left(\alpha^{3}+2 \alpha-c^{3}-\alpha c^{2}+\alpha^{2} c+2 c\right)\right\}
\end{array}\right.
$$

Thus, the solutions for $u(x, t)$ and $v(x, t)$ are obtained as follows

$$
\begin{array}{lrl}
u_{1}(x, t)=i(\alpha+c) \tanh (c t-\alpha x) & u_{2}(x, t)=-(\alpha+c) \operatorname{sech}(c t-\alpha x) \\
v_{1}(x, t)=\alpha^{2}-c^{2}+2 c(\alpha+c) \tanh ^{2}(c t-\alpha x)+\frac{1}{2} & v_{2}(x, t)=\frac{1}{2}\left(-\alpha^{2}+c^{2}\right) \\
& -2 c(\alpha+c) \operatorname{sech}^{2}(c t-\alpha x)+\frac{1}{2} \\
u_{3}(x, t)=-\frac{1}{2}(\alpha+c)(\operatorname{sech}(c t-\alpha x)-i \tanh (c t-\alpha x)) & \\
v_{3}(x, t)=\frac{1}{4}\left(2+(\alpha+c)\left(\alpha+\frac{4 i c}{\sinh (c t-\alpha x)-i}+c\right)\right) &
\end{array}
$$

Figure 2 shows selected solutions graphically. In the literature; different types of solutions related with Variant Boussinesq and Coupled Klein Gordon equations can be found $[10,14]$ and there in. However, within the knowledge of the authors, $u_{3}$ and $v_{3}$ solutions are new exact solutions of the considered equations. 


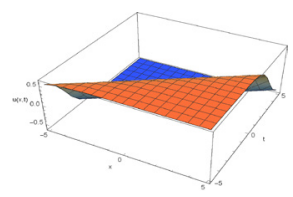

(a)

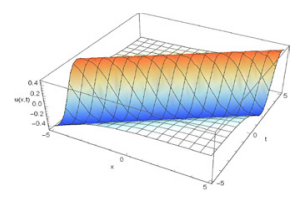

(c)

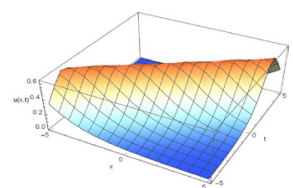

(b)

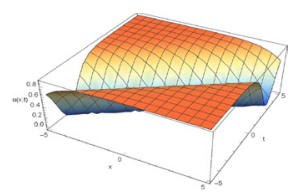

(d)

Figure 2: Solution of Coupled Klein equation for $\alpha=0.5, c=0.75$

\section{Conclusion}

The Sine-Gordon expansion method has been successfully applied to Variant Boussinesq equation and Klein Gordon equation. We have constructed the solutions in terms of hyperbolic functions. Also, the behavior of the newly obtained solutions are illustrated for some special values of the parameters. The method is quite efficient and practically well suited for use in obtaining the exact solutions of various partial differential equations.

\section{References}

[1] H. M. Baskonus, H. Bulut, Waves in Random and Complex Media, 26(4), 613-625, (2016)

[2] , M. J. Ablowitz, X. D. Luo, and Z. H. Musslimani, Journal of Mathematical Physics, 59.1: $011501 .(2018)$

[3] X. H. Zhao, B. Tian, X.Y. Xie, X.-Y. Wu, Y. Sun and Y.-J. Guo, Waves in Random and Complex Media, 28.2, 356-366,(2018)

[4] H. M. Baskonus, G. Yel, and H. Bulut, AIP Conference Proceedings, 1863: 1, 2017.

[5] M. T. Darvishi, S. Ahmadian, S. B. Arbabi,M. Najafi, Optical and Quantum Electronics 50.1:32,1-20,(2018)

[6] B. Zengin, H. Yaraneri, and S. Korunur, The European Physical Journal B 85.11, 388392, (2012).

[7] H. M.Baskonus, Nonlinear Dynamics, 86(1), 177-183, (2016)

[8] H. Bulut, T. A. Sulaiman and H. M. Baskonus, Optical and Quantum Electronics, 48, 12(564), 1-14, (2016)

[9] H. M. Baskonus, T. A. Sulaiman and H. Bulut,Optik- International Journal for Light and Electron Optics, 131, 1036-1043, (2017)

[10] K. Khan, M. A. Akbar, SpringerPlus, 3: 324, 1-17,(2014)

[11] H. M. Baskonus, T. A. Sulaiman, H. Bulut, Indian Journal of Physics,91(10),1237-1243,(2017)

[12] C. Cattani, T. A. Sulaiman, H. M. Baskonus, H. Bulut, European Physical Journal Plus, 133(228), 1-12, (2018)

[13] , M. L. Wang, Physics Letters A, 199:3-4, 169-172, (1995).

[14] M. Chen, Applied mathematics letters, 11.5, 45-49.(1998) 